\title{
ATMOSPHERIC LIMITS: A REVIEW OF THE EFFECT OF PATH LENGTH VARIATIONS ON THE COHERENCE AND ACCURACY OF VLBI
}

\author{
Alan E.E. Rogers \\ Haystack Observatory \\ Westford, MA 01886
}

\begin{abstract}
Interferometer phase fluctuations produced by path length variations in the troposphere limit the coherence to between 100 and 1000 seconds at a 3 millimeter wavelength. An expression for the coherence is given using an Allan variance to characterize the atmospheric phase fluctuations. Methods of optimizing the fringe detection threshold and calibrating the fringe visibilities under poor conditions of poor coherence are outlined. The astrometric and geodetic accuracy of VLBI is limited by our ability to calibrate the atmospheric path. Atmospheric "self-calibration" techniques which use the elevation dependence of interferometer delay to solve for the highly variable "wet" component of troposphere are discussed. Various models for the elevation dependence of atmospheric path or "mapping function" are reviewed. The accuracy limits imposed by the atmosphere are discussed.
\end{abstract}

\section{INTRODUCTION}

In early Very Long Baseline Interferometry (VLBI) experiments the phase fluctuations were largely due to instabilities in the frequency standards used to control the local oscillators. In more recent experiments at short wavelengths which used hydrogen maser frequency standards whose stability is $10^{-14}$ or better, the phase fluctuations are largely the result of atmospheric path variations. These path variations are non-dispersive and thus result in phase fluctuations which are proportional to the observing frequency. Experiments at wavelengths of 10 centimeters and longer start to be influenced by the ionospheric phase fluctuations which increase at a rate which is proportional to the observing wavelength. The atmospheric path length variations at wavelengths in the radio range are thought to be dominated by variations in the "wet" component or that due to water vapor which typically produces about 10 centimeters out of the approximately 2 meters of the total "wet" plus "dry" delay at the zenith. The "wet" component fluctuations, which are absent at optical wavelengths, constitute one of the few disadvantages of radio interferometry. However the development of Water Vapor

M. J. Reid and J. M. Moran (eds.), The Impact of VLBI on Astrophysics and Geophysics, 533-541.

(C) 1988 by the IAU. 
Radiometers (WVR) may eventually eliminate the problems of the "wet" component by providing accurate corrections for both the constant and time variable components of "wet" path delay.

\section{EXAMPLES OF ATMOSPHERIC PHASE FLUCTUATIONS}

Typical good weather interferometer phases are shown in Figure 1 below for an experiment at 3 millimeter wavelengths [Rogers et al, 1984].

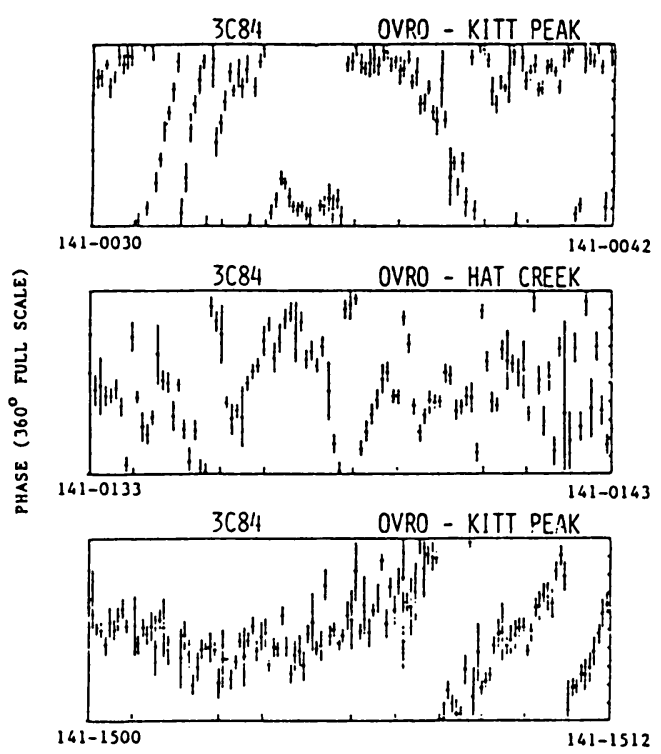

Figure 1. Typical $89-\mathrm{GHz}$ interferometer phases as a function of time from observations made in May 1982 using a 10.4-m antenna of the Owens Valley Radio Observatory (OVRO), a 6-m antenna of the Hat Creek Observatory and the 12-m antenna of the National Radio Astronomy Observatory on Kitt Peak. Scans are 12 min long with phases shown for 5-s segments.

The typical r.m.s. path length variations are about 1 millimeter for coherent averaging time of 5 seconds. These variations result in a significant loss of coherence for longer coherent integration as shown in Figure 2. The range of coherence loss for experiments of good weather observing is typically about 4 to 1 , the very best scans allowing a coherent integration of up to 700 seconds without large coherence losses. 


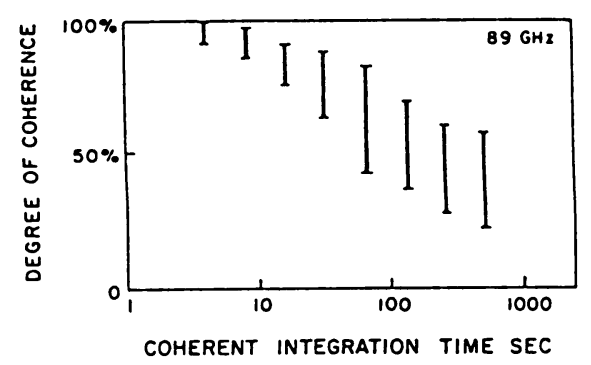

Figure 2. The range of normalized correlation coefficient as a function of coherent integration time for observations at $3 \mathrm{~mm}$ wavelength [Rogers et al, 1984].

\section{COHERENCE AND ALLAN STANDARD DEVIATION}

The coherence time $\mathrm{T}$ at observing frequency $\omega$ (radians/sec) and Allan standard deviation $\sigma(T)$ is approximately given by

$$
\omega T \sigma(T)=1
$$

A more precise relation has been developed by Rogers and Moran [1981] who starting with the following definition of a coherence function $\mathrm{C}(\mathrm{T})$

$$
C(T)=\left|\frac{1}{T} \int_{0}^{T} \exp [i \varphi(t)] d t\right|
$$

and using the method outlined by Rutman [1978] derived the following integral equation

$$
\begin{aligned}
\left\langle C^{2}(T)\right\rangle^{1 / 2}=\left[\frac { 2 } { T } \int _ { 0 } ^ { T } \operatorname { e x p } \left[-\frac{\omega^{2} \tau^{2}}{4}\left(\sigma^{2}(\tau)\right.\right.\right. & \\
& \left.\left.\left.+\sigma^{2}(2 \tau)+\cdots\right)\right](1-\tau / T) d \tau\right]^{1 / 2}
\end{aligned}
$$

Evaluation of the integral is simple in the case of white phase noise (Allan standard deviation power law exponent $=-1$ ) and white frequency noise (exponent $=-0.5$ ) resulting in 15 and 8 percent coherence loss when

$$
\omega \sigma(T) T=1
$$

The integral was also numerically evaluated to derive the coherence at several frequencies and integration times using the Allan standard deviation estimates 
for atmospheric phase fluctuations. The results are not significantly different, however, from the simple relationship derived from equation 1 which gives the maximum observing frequency for a coherence loss of approximately 10 to $20 \%$ as follows:

$$
\omega_{\max } \simeq 1 /(\sigma(T) T) \mathrm{rad} / \mathrm{s}
$$

This simple relation predicts a maximum observing frequency of $100 \mathrm{GHz}$ for a coherent integration time of 16 seconds and Allan standard deviation of $10^{-13}$. Allan standard deviation values derived from VLBI data [Rogers et al, 1984] along with the Allan standard deviation for the hydrogen maser is shown in Figure 3 below.

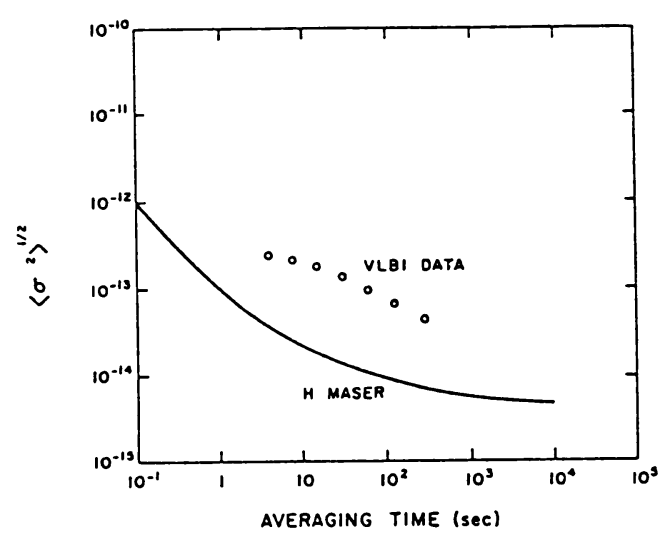

Figure 3. Allan standard deviation of atmospheric path length variation for an average of high SNR scans in April 1983. The solid curve is the Allan standard deviation observed between the Smithsonian Astrophysical Observatory (model VLG10) and Goddard Space Flight Center (model NP3) maser during tests in 1975 at Haystack Observatory. The masers used in the millimeter experiments were either of more recent design or upgraded versions for which the curve shown is a very conservative estimate of performance. (Allan standard deviation is given for difference between two sites and should be reduced by $2^{1 / 2}$ for path variations to a single site.)

\section{ANALYZING DATA WITH SEVERE PHASE FLUCTUATIONS}

In order to reduce the effects of phase noise on the estimation of correlation amplitude and closure phase, special data averaging methods are used. For a single baseline, the correlation amplitude is estimated by segmenting an observation. In this case the amplitude can be estimated by incoherently averaging $N$ segments. An estimate of the correlation amplitude is given by Moran [1976], as 


$$
A=\left[\frac{1}{N} \sum_{i=1}^{N} a_{i}^{2}-2\right]^{1 / 2}
$$

where $a_{i}$ is coherent amplitude or signal to noise ratio of each segment in units of the sigma noise level. The subtraction of 2 is needed to remove the bias that results from the noise. As long as the phase variations of the signal within each segment are small, the amplitude A will not be significantly reduced. Thus it is desirable to make the segments as short as possible to contain all the fringe energy while maintaining a reasonable signal to noise ratio for $A$. When the SNR for each segment is much greater than one, the SNR of A increases with the square root of $\mathrm{N}$. In this case the incoherent averaging produces no significant loss in SNR when compared wth coherent averaging. When the SNR of each segment is much less than one, a threshold proportional to the fourth root of $\mathrm{N}$ sets the detection limit. In this case the incoherent averaging process becomes relatively inefficient because the SNR will decrease with a reduction in segment length.

If fringes are detected on two baselines of a triangle, the coherent integration time can be extended on the third baseline by using the phases on the two stronger baselines to take out the phase variations. This technique requires a correction for the amplitude on the third baseline be made for phase noise on the two stronger "phase reference" baselines. The loss factors are multiplicative, or add when measured in decibels. The loss factor (Rogers el al. [1984]) depends on SNR and is greater than $0.9($ loss $<10 \%)$ for SNR $>2.5$ for each data segment.

\section{STATISTICAL MODELS OF PHASE FLUCTUATIONS}

Important statistical properties of the phase fluctuations are the r.m.s. variation with baseline length, source elevation (or path length) and time scale (Allan standard deviation). Armstrong and Sramek [1982] and Treuhaft and Lanyi [1987] have shown how the power law exponents are related for these statistical quantities assuming that "frozen" turbulence is moving over the observing sites with the wind. 


\begin{tabular}{|c|c|c|c|c|}
\hline Statistical function & model & value & meas. & Reference \\
\hline Spatial Power Spectrum(short) & $-\alpha$ & -3.67 & & \\
\hline Spatial Power Spectrum (long) & $-\alpha$ & -2.67 & & \\
\hline r.m.s. vs baseline (short) & $\alpha-2$ & 1.67 & 1.4 & $\begin{array}{l}\text { Armstrong and } \\
\text { Sramek [1982] }\end{array}$ \\
\hline (long) & $"$ & 0.67 & 0 & see text \\
\hline Allan standard dev. $(\tau<30)$ & $(\alpha-4) / 2$ & -0.17 & -0.25 & $\begin{array}{l}\text { Rogers et } \\
\text { al. [1981] }\end{array}$ \\
\hline$"(\tau>30)$ & $"$ & -0.67 & -0.50 & $"$ \\
\hline$" \quad$ (long) & $"$ & -0.67 & -0.63 & $\begin{array}{l}\text { Hogg et } \\
\text { al.[1981] }\end{array}$ \\
\hline$" \quad($ short $)$ & $"$ & -0.17 & -0.07 & $\begin{array}{r}\text { Thompson et } \\
\text { al.[1975] }\end{array}$ \\
\hline (long) & $"$ & -0.67 & -0.54 & $"$ \\
\hline r.m.s. vs path length & & 0.50 & 0.50 & $\begin{array}{l}\text { Treuhaft and } \\
\text { Lanyi [1987] }\end{array}$ \\
\hline
\end{tabular}

\section{TABLE OF POWER LAW EXPONENTS}

The table above shows the predicted and measured power law exponents for a model of Kolmogorov turbulence with two-dimensional spatial power exponent range of $-11 / 3$ for scales shorter than the atmospheric scale height and $-8 / 3$ for longer scales. The value of $-11 / 3$ corresponds to fully developed isotropic turbulence while $-8 / 3$ is the value expected for larger scales for which the structure effectively becomes two dimensional. The r.m.s. phase and phase rate variation with source elevation should depend on the square root of the path length at least for scales shorter than the scale height. Measured values of the power laws agree fairly well with the theory except that the theory is not adequate to correctly characterize the very long scales and correctly predict the observed independence of the r.m.s. fluctuations with baseline length for baselines longer than about 100 $\mathrm{km}$. A "saturation" scale length like that introduced introduced by Treuhaft and Lanyi [1987] is needed to refine the model.

The strength of the phase fluctuations or magnitude of the turbulence varies (at 10 seconds) from an Allan standard deviation of $10^{-14}$ under the best meteorological conditions to $10^{-12}$ under the worst conditions. Most of the phase variations are thought to be due to the "wet" component but while Resch et al [1984] show encouraging results in their experiment to use a WVR to correct interferometric phases at the VLA it seems probable that perhaps as much as $30 \%$ of the variations are from the dry component. Some experiments are planned in which both the wet and dry components can be measured along a line from the site to an aircraft using multi-wavelength optical and radio transponders. 


\section{TROPOSPHERIC PATH DELAY MODELS}

While the atmospheric path variations discussed in the previous sections limit the coherent integration and add noise to the measurements an incomplete knowledge of the average atmospheric path over the coherent integration period will limit the accuracy of the astrometric and geodetic parameters estimated from measurements of phase and group delay. In this section I review the path delay models.

The "dry" component of path delay at the zenith in a static atmosphere depends only on the ground pressure. The path delay variation with elevation $\epsilon$ or "mapping" fuction $m(\epsilon)$ can be predicted by a "ray trace" through an atmospheric model. A simple model for the atmospheric being one made of spherical shells of gas of known constituents and refractivity obeying the gas equations and in hydrostatic equilibrium. Additional model parameters are the ground temperature, lapse rate and height of the tropospause. For many years two simple analytic approximations to the ray tracing results were used in the analysis of VLBI data. The simplest model was that of Chao [1972].

$$
m(\epsilon)=1 /[\sin \epsilon+[0.00143 /(\tan \epsilon+0.0445)]]
$$

and contains only fixed constants for "average" conditions including an average contribution from the "wet" component. A somewhat more sophisticated model was that of Marini [1974] (and modified for the radio range of frequencies in an unpublished note)

$$
m(\epsilon)=1 /[\sin \epsilon+[(B /(A+B)) /(\sin \epsilon+0.015)]]
$$

whose constants $\mathrm{A}$ and $\mathrm{B}$ are dependent on ground measurements of pressure, temperature and humidity. After it was suspected that neither of these models were quite good enough Davis et al [1985] developed a model known as CfA-2.2 as follows:

$$
\begin{gathered}
m(\epsilon)=1 /[\sin \epsilon+[a /(\tan \epsilon+[b /(\sin \epsilon-0.009)])]] \\
\text { where } \mathrm{a}=0.001185+\text { correction terms } \\
\text { and } \mathrm{b}=0.001144+\text { correction terms. }
\end{gathered}
$$

The terms added to $\mathrm{a}$ and $\mathrm{b}$ are linearized corrections for the deviations of the partial pressure of water vapor, ground temperature, pressure, atmospheric lapse rate and height of the tropopause from the nominal average values for which the constants $\mathrm{a}$ and $\mathrm{b}$ are valid without the added terms.

CfA-2.2 fits the ray tracing results to within $2 \mathrm{~mm}$ down to 5 degrees elevation whereas the simpler functional forms of Chao and Marini deviate from the numerical ray tracing by several centimeters or more at elevations below 15 degrees. 


\section{TESTS OF THE ACCURACY OF ATMOSPHERIC MODELS}

Errors in the atmospheric model will result, primarily, in an error in the estimate of the local station height derived from inteferometric delays to a reference point whose path delay has been accurately corrected. For long baselines the error in height or radial component of either site $\Delta r$ leads to an error in baseline length of

$$
\Delta b \simeq \frac{b \Delta r}{2 r_{e}}
$$

where $r_{e}$ is the radius of the earth. The first suggestion of the significant errors in the Chao and Marini models came from a test made by Davis et al [1985] of the dependence of baseline length on the elevation cutoff used in the least squares analysis. The elevation cutoff test showed a baseline length change of about $0.5 \mathrm{~cm}$ per $1000 \mathrm{~km}$ (fractional error of $5 \times 10^{-9}$ ) for a change in elevation cutoff between 5 and 15 degrees. The use of CfA-2.2 has made changes in baseline length with elevation cutoff undetectable at the present level of measurement uncertainty. With the improved atmospheric models the repeatability of baseline length measurements are at the few centimeter level for baselines longer than $3000 \mathrm{~km}$, about 1 centimeter for intermediate baselines and at the millimeter level for baselines under $300 \mathrm{~km}$. However even with the improved "mapping" function it is still necessary to either solve for the zenith path delay at each site every few hours, estimate the zenith path continuously using Kalman filter analysis or make highly accurate pressure and WVR measurements at each site to calibrate the atmospheric path.

\section{SUMMARY}

Atmospheric phase fluctuations are present at the level of approximately $10^{-13}$ (Allan standard deviation) and limit coherent integration of $3 \mathrm{~mm}$ VLBI to a few hundred seconds. These fluctuations are independent of baseline length for baselines longer than $100 \mathrm{~km}$ and increase with the square root of the path length thereby being approximately doubled at 15 degrees elevation. Accurate models for the elevation dependence of the atmospheric path length are needed to remove systematic errors in astrometric and geodetic results in order to reach a fractional accuracy of better than $5 \times 10^{-9}$.

\section{References}

Armstrong, J.W., and R.A. Sramek, 'Observations of tropospheric phase scintillations at $5 \mathrm{GHz}$ on vertical paths', Radio Sci., 17, 1579-1586, 1982. 
Chao, C. C., 'A model for tropospheric calibration from daily surface and radiosonde balloon measurements.' Tech. memo Cal. Inst. technol. Jet Propulsion Lab., 391-350, 1972.

Davis, J. L., T. A. Herring, I. I. Shapiro, A. E.E. Rogers, and G. Elgered, 'Geodesy by radio interferometry: Effects of atmospheric modeling errors on estimates of baseline length', Radio Sci., 20, 1593-1607, 1985.

Hogg, D. C., F. O. Guiraud, and W. B. Sweeney, 'The short-term temporal spectrum of precipitable water vapor', Science,213, 1112-1113, 1981.

Moran, J. M., 'Very long baseline interferometric observations and data reduction', in Methods of Experimental Physics, edited by M. L. Meeks, vol. 12, part C, pp. 228-260, Academic, New york, 1976.

Marini, J. W., 'Correction of satellite tracking data for an arbitrary tropospheric profile', Radio Sci.,7, 223-231, 1972. and unpublished note 1974.

Resch, G. M., D. E. Hogg, and P. J. Napier, 'Radiometeric correction of atmospheric path length fluctuations in interferometric experiments', Radio Sci.,19, 411-422, 1984.

Rogers, A. E.E., A. T. Moffet, D. C. Backer, and J. M. Moran, 'Coherence limits in VLBI observations at 3-millimeter wavelength', Radio Sci.,19, 1552-1560, 1984.

Rogers, A. E.E., and J. M. Moran, 'Coherence limits for very-long-baseline interferometry', IEEE Trans. Instrum. Meas., IM-30(4), 283-286, 1981.

Rutman, J., 'Characterization of phase and frequency instabilities in precise frequency sources: Fifteen years in progress', Proc. IEEE,66, 1048-1075, 1978.

Thompson, M. C., L. E. Wood, H. B. James, D. Smith, 'Phase and amplitude scintillations in the 10 to $40 \mathrm{GHz}$ band', IEEE Trans. Antennas Propag.,AP-23, 792-797, 1975.

Treuhaft, R.N. and G.E. Lanyi, 'The effect of the dynamic wet troposphere on radio interferometric measurements', Radio Sci., 22, 251-265, 1987. 